\title{
Phase Transition in Quasi-Two-Dimensional Horizontally Driven Granular System
}

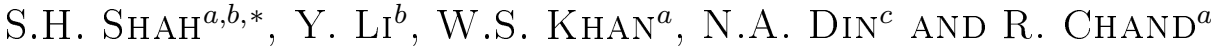 \\ ${ }^{a}$ Physics Department, Beijing Institute of Technology, 100081 Beijing, China \\ ${ }^{b}$ Institute of Physics, Chinese Academy of Sciences, 100190 Beijing, China \\ ${ }^{c}$ Physics Department, Gomal University D.I. Khan, Pakistan
}

(Received March 14, 2011; in final form January 18, 2012)

\begin{abstract}
In an experimental setup, consisting of quasi-two-dimensional square cell, the motion of monodisperse spherical steel balls is studied. The cell is vibrated horizontally to eradicate the compaction of balls due to gravity. By raising the number density of the steel balls, the dilute fluid has been gradually alternated into dense fluid. The temperature of granular media, along the driven and transverse directions, has been found to be anisotropic. Transition from granular gas to the liquid-like fluid has been observed. The radial distribution function of the balls in the cell is investigated for the authentication of the structural changes. Furthermore, the fast Fourier transformation for the $2 \mathrm{D}$ system is carried out to confirm these changes.
\end{abstract}

PACS: 81.05.Rm, 64.60.-i

\section{Introduction}

Granular materials have been of fundamental interest for scientists and engineers because they show behaviour redolent to conventional phases of matter [1-3]. For example, the sand can flow like a fluid, under certain conditions. The experimental and theoretical studies of dilute granular systems show that they are dissipative in nature; hence their motions stop after some time [2-5]. However, to reach their steady state and prevent inelastic collapse, energy must be regularly provided to them from some external source [6-8]. Under the typical conditions of external excitation intensity and volume fraction of system, the granular materials can behave like a solid and resists to the external shearing. However, they can flow like liquids, and in dilute phase can behave like gases. Due to the complex behaviour in these states it can be claimed that the granular matter is distinct state of matter and has its own rights in addition to the liquids, gases and solids $[9,10]$. The granular materials found in nature are macrosized, it means that the thermal fluctuations do not play any role in their motion and regular thermodynamic temperature is immaterial in this regard.

The inter-grain interactions are dissipative due to the dissipative nature of collisions between the colliding particles. In the dry granular media, the interactions between the grains are only through the repulsive contact forces. The outcome of a collision between two moving grains will be the part of their energy lost and transferred to other forms, for example grains thermal energy, plastic deformations and microcracks formations [11, 12]. If we do not supply energy from some external source to

\footnotetext{
* corresponding author; e-mail: sajjadjeeven2@yahoo.com
}

the granular system, the kinetic energy of the system is lost quickly and finally all the grains will come to rest due the effect of gravity. The steady fluidised state is possible only with a continuous supply of energy. However, supplying a continuous energy from some external source, the system can be fluidized and it can exhibit nearly all known hydrodynamic behaviours and instabilities.

In the experiments, the energy can be provided from external source to the granular media through the boundary walls of the container i.e. by driving the walls of the container. In some simulations [10, 13] and mostly in the analytic theories, this heating or driving has been carried out uniformly through the whole container (uniform heating), and all of the gas particles are being driven individually by a white-noise source. However, in case of experiments of granular gases, usually energy is provided to the granular gas through shaking or vibrations of the walls of the container.

Although the energy is being continuously supplied, the fluid develops a gradient in density $[14,15]$. In most of the experiments, the particles are vibrated vertically [16-18]. However, in vibrating the system vertically, there may be the particle's compaction caused by the pull of gravity and it can introduce errors in the results. To eradicate the effect of gravity pull on the particles, we have designed an experimental setup in which the particles can be vibrated horizontally. Hence in this setup, for different particle densities, it will be interesting to know about the existence of different granular phases under external driving at steady state.

\section{Experimental setup}

We are using a square cell of aluminium having internal dimensions of $40 \mathrm{~mm} \times 40 \mathrm{~mm}$. The monodisperse 
stainless steel spherical balls, each with a diameter of 2 $\mathrm{mm}$, are brought into play throughout the experiment. To make the cell quasi-two-dimensional, the depth of the cell is taken to be $2.35 \mathrm{~mm}$. Each layer of balls in the cell comprise of 20 balls. This cell has a firmly attached glass base and a detachable glass lid. The cell is mounted on an oily platform, upon which it can move very easily. An electromagnetic oscillator is connected to this cell, which can vibrate the cell at different frequencies. This whole arrangement is fixed on a table provided with fine pitch screws, which can be used for the levelling of the whole arrangement.

Monodisperse stainless steel spherical balls are placed in the cell and are vibrated horizontally along a direction parallel to $x$-axis. The main source of energy inputs is the two boundaries, which are perpendicular to the direction of vibration. The balls are illuminated from above to produce a small bright spot at the top of each of them. These spherical balls are viewed at a position vertically above the cell, through a fast DALSA-1 Camera with 955 frames/second and bearing spatial resolution of 256 pixels $\times 256$ pixels. For the experiment the cell vibrations are sinusoidal and the frequency of vibrator is fixed to be $\nu=44.33 \mathrm{~Hz}$. The maximum acceleration is $A \omega^{2}$, where $\omega=2 \pi \nu$ is the angular frequency and $A$ is vibration amplitude. The amplitude of vibrations is 2 $\mathrm{mm}$ and is also kept constant throughout the whole experiment. The schematic illustration of the experimental setup is given in Fig. 1.

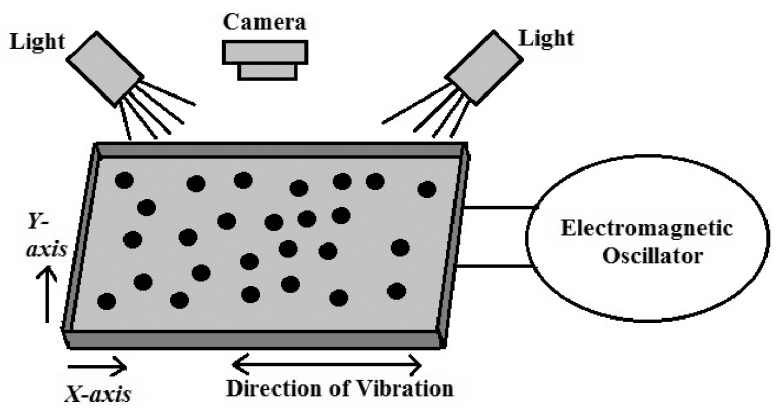

Fig. 1. Schematic diagram of experimental setup. The stainless steel balls each having diameters $2 \mathrm{~mm}$ are placed inside square cell of aluminium with internal area $40 \mathrm{~mm} \times 40 \mathrm{~mm}$ and a depth of $2.35 \mathrm{~mm}$. A camera of spatial resolution 256 pixels $\times 256$ pixels, mounted vertically above the cell can take the photographs. The cell can vibrate along $x$-axis.

\section{Results and discussions}

The surface of the cell was accurately levelled with the aid of regulating fine pitch screws, to within $\pm 0.005^{\circ}$. If there is no proper horizontal level, it may cause clustering of particles, which can affect the results.

The experiment was started with two numbers of layers i.e. 40 balls. The balls are placed in the cell and are vibrated sinusoidal along $x$-axis at constant frequency of
$44.33 \mathrm{~Hz}$. Initially the system is homogeneous and does not have gradient in density, shown in Fig. 2a. When we vibrate the cell, there are two kinds of collisions i.e. ballboundary collisions and ball-ball collisions. The ballboundary collisions are the origin of energy injected into the system whilst during ball-ball collisions, the balls dissipate energy. We increase the number of balls in the cell in the steps of one layer. As we increase the number of balls, clustering starts inside the cell. The reason is that the balls are compressed in the middle of the cell, due to the pressure of those balls which are running in after colliding from the boundaries. The balls in the denser areas of clusters collide more frequently as compared to that in the dilute area. There is strong energy dissipation in denser areas, so the denser areas cool faster as compared to dilute areas. A gradient of pressure is developed which fluxes more number of balls into the denser area. As a result, the ball-ball collision rate and the dissipation of energy are further increased causing strong clustering and compaction.
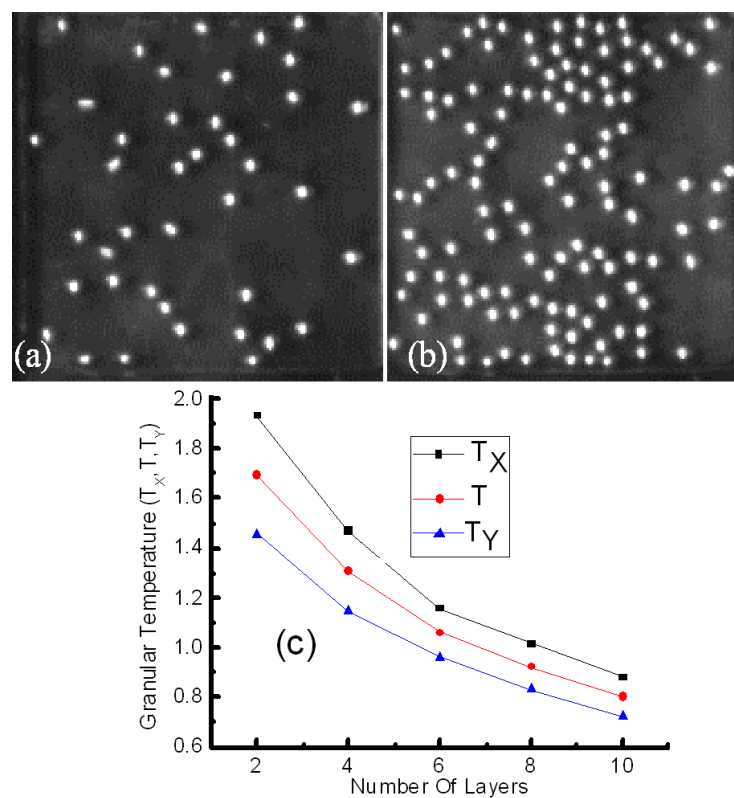

Fig. 2. (a), (b) Snapshots from the experiment: (a) for two layers of balls there is almost uniform density in the middle and boundary of the cell, (b) for six layers there is clustering of balls, these clusters are mobile, and in the figure they are moving towards left end of the cell. (c) The granular temperature as a function of number density. The black squares show the granular temperature $T_{x}$, red circles for $T$ and blue triangles for $T_{y}$. For simplicity, only the even number values are shown.

When the clusters are smaller, they are vastly mobile and assemble and disperse, while they move in the container [19, 20]. Also sometimes the high velocity balls coming in from the boundaries can disturb them. But as the clusters grow up in size, further they cannot be destabilized by the high velocity balls, shown in Fig. $2 \mathrm{~b}$. 
As the real granular materials are inelastic, hence the energy inputs from the boundaries is not enough to break the strong clusters and thermalize the system.

When we increase the number density, the mean free path and temperature decreases but this decrease is nonlinear, shown in Fig. 2c. We have also calculated the values of granular temperature $T_{X}, T_{Y}$ and $T$, where $T=\frac{1}{2}\left(T_{X}+T_{Y}\right)$. The values of $T_{X}, T_{Y}$ and $T$, plotted as a function of number density are shown in Fig. 2c. The $T_{X}$ and $T_{Y}$ are found to be anisotropic $T_{X} \neq T_{Y}$ i.e. $\left\langle v_{x}^{2}\right\rangle \neq\left\langle v_{y}^{2}\right\rangle$. Also we have found in our experiment that $T_{X}>T>T_{Y}$. It shows the non-equipartition of energy in granular media.

The above discussion shows that on increasing number density, the mean free path and the granular temperature is decreased causing clustering which leads to condensation. To avoid the effects from transverse boundaries, only the middle portion of the cell is chosen for calculations, whose area is $13.3 \mathrm{~mm} \times 40 \mathrm{~mm}$ and this equals one third of the total area of cell. To interlink this macroscopic behaviour with the structural configuration of the spherical steel balls, the radial distribution function $\rho(r)$ [20] is calculated for this central portion of the cell i.e.

$$
\rho(r)=C(r)\left\langle\sum_{i} \sum_{i \neq j} \delta\left(r-r_{i, j}\right)\right\rangle
$$

Here $r_{i, j}$ is the distance between the $i$-th and $j$-th steel ball and $C(r)$ is normalization constant.

Furthermore, the packing fraction for the middle portion of the cell is computed by using

$$
\Psi=\mathrm{N} \phi / \mathrm{XY},
$$

where $N$ is the number and $\phi$ is the two-dimensional projected area of steel balls, $X$ and $Y$ are the longitudinal and transverse dimensions of the cell.

Figure 3a shows curve of $\rho(r)$ for less than ten layers in the cell. Here $\rho(r)$ has a single peak, pointed out by the dashed vertical line, at $r / D=1$ which rapidly decreases at large distances, as anticipated for disordered gases. Here, $D$ is the diameter of the ball.

Figure 3b shows curve of $\rho(r)$ for ten layers in the cell. Here $\rho(r)$ shows peaks, pointed out by vertical dashed lines, at $r / D=1$ and 2 ball diameters and it exhibits liquid-like behaviour. The surrounding spheres exhibit correlations in their positions and the maxima might be affiliated with concentric shells of neighbours. The hard sphere liquids commonly show such behaviour. Also the corresponding packing fraction, for ten layers of balls in the cell, is found to be $\Psi=0.5$. To investigate any developed structural change, the fast Fourier transformation (FFT) in two dimensions is performed and is shown in Fig. 3c. For the filling fraction of 0.5 , the configuration of grains does not show any specific structure but displays a continuous intensity distribution in circles of different radii.

It means that we have a gas like structure below ten layers and a liquid-like structure at ten layers. Hence,

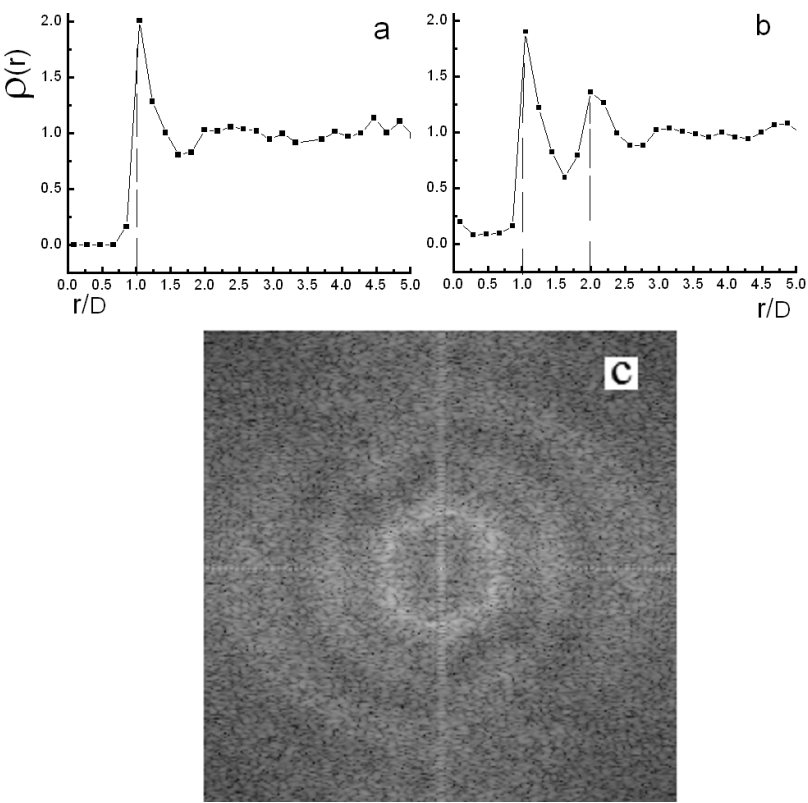

Fig. 3. (a) The radial distribution function for less than ten layers and there is a single peak at about $r / D=1$ exhibiting the disordered gases structure. (b) The radial distribution function for ten number of layers and there are two peaks at about $r / D=1,2$ exhibiting liquid like behaviour. The vertical dashed lines indicate the expected position of the peaks in each of the phases. (c) FFT of the fluid corresponding to the filling fraction of 0.5 .

there is transition of phase from gas to liquid when we reach ten layers whereas the corresponding packing fraction is 0.5 .

\section{Conclusion}

We come to the conclusion that in the absence of gravity induced compaction of balls, an increase in the number of particles in the cell results in a corresponding increase in the dissipation of energy. Hence, as a result the clusters are formed, which lead to produce a dense fluid. However the temperature of the fluid along the direction of vibration and in the transverse direction is not the same. At sufficient number density of balls, there occurs a change in phase from gas-like granular fluid to the liquid-like granular fluid.

\section{Acknowledgments}

This work is supported by National Natural Science Foundation of China (grant nos. 10720101074 and 10874209), and the Chinese Academy of Sciences (grant nos. KKCX1-YW-03 and KJCX2-YW-L08).

\section{References}

[1] I. Goldhirsch, G. Zanetti, Phys. Rev. Lett. 70, 1619 (1993). 
[2] I. Goldhirsch, M. L. Tan, G. Zanetti, J. Sci. Comput. 8, 1 (1993).

[3] S. McNamara, W. R. Young, Phys. Rev. E 50, R28 (1994).

[4] W. Losert, D. G. W. Cooper, J.P. Gollub, Phys. Rev. E 59, 5855 (1999).

[5] J.S. Olafsen, J.S. Urbach, Phys. Rev. Lett. 81, 4369 (1998).

[6] D.L. Blair, A. Kudrolli, Phys. Rev. E 64, 050301 (2001).

[7] H.M. Jaeger, S.R. Nagel, Science 255, 1523 (1992).

[8] P.G. de Gennes, Rev. Mod. Phys. 2, S374 (1999).

[9] H.M. Jaeger, S.R. Nagel, R.P. Behringert, Rev. Mod. Phys. 68, 1259 (1996).

[10] R.M. Iverson, Rev. Geophys. 35, 245 (1997).

[11] I. Goldhirsch, Ann. Rev. Fluid Mech. 35, 267 (2003).
[12] P.G. de Gennes, Rev. Mod. Phys. 71, 374 (1999).

[13] D.R.M. Williams, F.C. Mackintosh, Phys. Rev. E 54, R9 (1996).

[14] S.J. Moon, M.D. Shattuk, J.B. Swift, Phys. Rev. E 64, 031303 (2001).

[15] A. Barrat, E. Trizac, Phys. Rev. E 66, 051303 (2002).

[16] E.L. Grossman, T. Zhou, E. Ben-Naim, Phys. Rev. E 55, 4200 (1997).

[17] F. Rouyer, N. Menon, Phys. Rev. Lett. 85, 3676 (2000).

[18] J.S. Vanzon, F.C. Mackintosh, Phys. Rev. Lett. 93, 038001 (2004).

[19] I. Goldhirsch, G. Zanetti, Phys. Rev. Lett. 70, 1619 (1993).

[20] P.M. Reis, G. Ehrhart, A. Stephenson, Euro Phys. Lett. 66, 357 (2004) 